\title{
RESISTIVE-WALL IMPEDANCE OF AN INFINITELY LONG MULTI-LAYER CYLINDRICAL BEAM PIPE
}

\author{
E. Métral, B. Salvant ${ }^{1)}$, B. Zotter \\ CERN, Geneva, Switzerland
}

\begin{abstract}
The resistive-wall impedance of cylindrical vacuum chambers was first calculated more than forty years ago under some approximations. Since then many papers have been published to extend its range of validity. In the last few years, the interest in this subject has again been revived for the LHC graphite collimators, for which a new physical regime is predicted. The first unstable betatron line in the LHC is at $8 \mathrm{kHz}$, where the skin depth for graphite is $1.8 \mathrm{~cm}$, which is smaller than the collimator thickness of $2.5 \mathrm{~cm}$. Hence one could think that the resistive thick-wall formula would be about right. It is found that it is not, and that the resistive impedance is about two orders of magnitude lower at this frequency, which is explained by the fact that the skin depth is much larger than the beam pipe radius. Starting from the Maxwell equations and using field matching, a consistent derivation of both longitudinal and transverse resistivewall impedances of an infinitely long cylindrical beam pipe is presented in this paper. The results, which should be valid for any number of layers, beam velocity, frequency, conductivity, permittivity and permeability, have been compared to previous ones.
\end{abstract}

${ }^{1)}$ EPFL, Lausanne, Switzerland

CERN, 


\title{
RESISTIVE-WALL IMPEDANCE OF AN INFINITELY LONG MULTI-LAYER CYLINDRICAL BEAM PIPE
}

\author{
E. Métral, B. Zotter (CERN, Geneva) and B. Salvant (EPFL, Lausanne)
}

\begin{abstract}
The resistive-wall impedance of cylindrical vacuum chambers was first calculated more than forty years ago under some approximations. Since then many papers have been published to extend its range of validity. In the last few years, the interest in this subject has again been revived for the LHC graphite collimators, for which a new physical regime is predicted. The first unstable betatron line in the LHC is at $8 \mathrm{kHz}$, where the skin depth for graphite is $1.8 \mathrm{~cm}$, which is smaller than the collimator thickness of $2.5 \mathrm{~cm}$. Hence one could think that the resistive thick-wall formula would be about right. It is found that it is not, and that the resistive impedance is about two orders of magnitude lower at this frequency, which is explained by the fact that the skin depth is much larger than the beam pipe radius. Starting from the Maxwell equations and using field matching, a consistent derivation of both longitudinal and transverse resistivewall impedances of an infinitely long cylindrical beam pipe is presented in this paper. The results, which should be valid for any number of layers, beam velocity, frequency, conductivity, permittivity and permeability, have been compared to previous ones.
\end{abstract}

\section{INTRODUCTION}

Renewed interest in this old subject [1] is due to the important role of resistive-wall effects for collective beam stability in large circular proton accelerators or colliders such as the LHC. In particular, the numerous collimators in the machine are made of highly resistive graphite to withstand the high temperatures generated by the impact of high-energy protons, and will be moved into positions very close to the beam to protect the surrounding superconducting magnets from stray protons. These devices could create such high impedances as to severely limit the beam current and hence the performance of the collider.

A number of papers have been published on this subject in the last few years $[2,3,4]$, which were already discussed in Ref. [5]. A more recent one was published in Ref. [6]. In this report, whose transverse part is a summary of Refs. [7,8] and whose results were already briefly discussed in Ref. [9], the exact calculation of the electromagnetic fields excited by an infinitesimally thin, annular particle beam of finite radius in a surrounding coaxial vacuum chamber of infinite length is described. The method used to compute the fields is reviewed in the first section, while the longitudinal and transverse impedances are discussed in the following sections. It is worth mentioning that the "low-frequency" regime, which is of primary importance for the LHC collimators, was already obtained in Ref. [10] under some approximations.

\section{FIELD CALCULATIONS}

Starting from Maxwell equations, assuming that in the frequency domain all the field quantities are proportional to $e^{j \omega t}$ (which will be omitted below for simplicity), and combining the conduction and displacement current terms, yields the following scalar Helmholtz equations for the longitudinal field components in the circular cylindrical coordinates $(r, \theta, z)$

$$
\begin{aligned}
& {\left[\frac{1}{r} \frac{\partial}{\partial r}\left(r \frac{\partial}{\partial r}\right)+\frac{1}{r^{2}} \frac{\partial^{2}}{\partial \theta^{2}}+\frac{\partial^{2}}{\partial z^{2}}+\omega^{2} \mu \varepsilon_{c}\right] H_{z}} \\
& =\left[\frac{1}{r} \frac{\partial v_{r}}{\partial \theta}-\frac{\partial\left(r v_{\theta}\right)}{\partial r}\right] \rho, \\
& {\left[\frac{1}{r} \frac{\partial}{\partial r}\left(r \frac{\partial}{\partial r}\right)+\frac{1}{r^{2}} \frac{\partial^{2}}{\partial \theta^{2}}+\frac{\partial^{2}}{\partial z^{2}}+\omega^{2} \mu \varepsilon_{c}\right] E_{z}} \\
& =\frac{1}{\varepsilon} \frac{\partial \rho}{\partial z}+j \omega \mu \rho v_{z} .
\end{aligned}
$$

Here, $H$ and $E$ are the magnetic and electric fields respectively, $\omega=2 \pi f$ is the angular frequency, $j$ is the imaginary unit, $\rho$ is the (source) charge density, $v$ is the source velocity, and

$$
\begin{gathered}
\varepsilon_{c}=\varepsilon_{0} \varepsilon^{\prime}=\varepsilon_{0} \varepsilon_{r}+\frac{\sigma}{j \omega}, \quad \varepsilon=\varepsilon_{0} \varepsilon_{r}, \\
\mu=\mu_{0} \mu^{\prime}=\mu_{0} \mu_{r}\left(1+j \tan \vartheta_{\mathrm{M}}\right),
\end{gathered}
$$

where $\varepsilon^{\prime}\left(\mu^{\prime}\right)$ is the relative complex permittivity (permeability) of the pipe with $\varepsilon_{0}\left(\mu_{0}\right)$ the permittivity (permeability) of vacuum and $\varepsilon_{r}\left(\mu_{r}\right)$ the relative permittivity (permeability) of the pipe, $\sigma$ is the electric conductivity, and $\tan \vartheta_{M}$ is the loss tangent.

The homogeneous equations can be solved by separation of variables, writing the longitudinal component of the magnetic and electric fields as $\Theta(\theta) Z(z) R(r)$. It is easily found that $\Theta(\theta)=e^{ \pm j m \theta}$, where $m$ is called the azimuthal mode number, and $Z(z)=e^{ \pm j k z}$, where $k=\omega / v$ is called the wave 
number. The function $R(r)$ is found by solving the following equation

$$
\frac{1}{r} \frac{d}{d r}\left(r \frac{d R}{d r}\right)-\left(\frac{m^{2}}{r^{2}}+v^{2}\right) R=0,
$$

with $v=k \sqrt{1-\beta^{2} \varepsilon^{\prime} \mu^{\prime}}$, which is called the radial propagation constant, where $\beta$ is the relativistic velocity factor. It is known that the solutions of the differential Eq. (5) are the modified Bessel functions of $m^{\text {th }}$ order and argument $v r$, called $I_{m}(v r)$ and $K_{m}(v r)$.

The source charge density $\rho$ has to be specified at this stage to obtain the exact forms of the azimuthal and axial functions $\Theta(\theta)$ and $Z(z)$. A macro-particle of charge $Q=N_{b} e$ is assumed to move along the pipe (in the $z$ direction) with an offset $r=a$ in the $\vartheta=0$ direction and with velocity $v=\beta c$ (equal to the bunch velocity $\left.v_{b}=\beta_{b} c\right)$. Neglecting betatron and synchrotron oscillations, assuming that the beam is not affected by the wake field, and using the azimuthal Fourier decomposition of the charge, the charge density can be written [11]

$$
\rho(r, \vartheta, z ; t)=\sum_{m=0}^{\infty} \frac{P_{m} \cos (m \vartheta)}{\pi a^{m+1}\left(1+\delta_{m 0}\right)} \delta(r-a) \delta(z-v t),
$$

where $P_{m}=Q a^{m}$ is the $m^{\text {th }}$ multipole moment, and $\delta_{m 0}=1$ if $m=0,0$ if $m \neq 0$. Proceeding to the frequency domain and considering only one mode $m$, it is seen that the charge density $\rho_{m}$ is proportional to $\cos (m \vartheta) e^{-j k z}$, which leads to the following longitudinal components of the electric and magnetic fields (reinserting the time dependence)

$$
\begin{aligned}
& H_{z}=\sin (m \theta) e^{j(\omega t-k z)}\left[C_{1} I_{m}(v r)+C_{2} K_{m}(v r)\right], \\
& E_{z}=\cos (m \theta) e^{j(\omega t-k z)}\left[C_{3} I_{m}(v r)+C_{4} K_{m}(v r)\right],
\end{aligned}
$$

where $C_{1,2,3,4}$ are constants to be determined by field matching, as all field strength components have to be matched, i.e. in the absence of surface charges and currents the four tangential field strengths have to be continuous (matching of the radial components is redundant).

Finally, using Maxwell equations in a source-free region, the transverse field components can be deduced from the longitudinal ones (with $\vec{G}=Z_{0} \vec{H}$, where $Z_{0}$ is the free-space impedance)

$$
\begin{aligned}
& E_{r 0}=\frac{j k}{v^{2}}\left(\beta \mu^{\prime} \frac{m G_{z 0}}{r}+\frac{d E_{z 0}}{d r}\right), \\
& E_{\theta 0}=-\frac{j k}{v^{2}}\left(\frac{m E_{z 0}}{r}+\beta \mu^{\prime} \frac{d G_{z 0}}{d r}\right), \\
& G_{r 0}=\frac{j k}{v^{2}}\left(\beta \varepsilon^{\prime} \frac{m E_{z 0}}{r}+\frac{d G_{z 0}}{d r}\right),
\end{aligned}
$$

$$
G_{\theta 0}=\frac{j k}{v^{2}}\left(\frac{m G_{z 0}}{r}+\beta \varepsilon^{\prime} \frac{d E_{z 0}}{d r}\right),
$$

where $E_{z} / E_{z 0}=E_{r} / E_{r 0}=G_{\vartheta} / G_{\vartheta 0}=\cos (m \vartheta)$ and $E_{\vartheta} / E_{\vartheta 0}=G_{r} / G_{r 0}=G_{z} / G_{z 0}=\sin (m \vartheta)$.

\section{LONGITUDINAL IMPEDANCE}

Considering only the term $m=0$ (longitudinal monopole), the charge density, in the frequency domain, is given by

$$
\rho_{0}(r, z ; \omega)=\frac{Q}{2 \pi a v} \delta(r-a) e^{-j k z} .
$$

The longitudinal source electric field in the vacuum region between the beam and the vacuum pipe ( $a \leq r \leq b$, where $b$ is the inner beam pipe radius) has been computed in Ref. [12] and is given by

$$
\begin{aligned}
& E_{z}^{(s)}(r, z ; \omega) \\
& =\frac{j \omega Q}{2 \pi \varepsilon_{0} v^{2} \gamma^{2}} I_{0}(s)\left[K_{0}(u)-\alpha_{\mathrm{TM}} I_{0}(u)\right] e^{-j k z},
\end{aligned}
$$

where $\gamma=\left(1-\beta^{2}\right)^{-1 / 2}$ is the relativistic mass factor, $s=k a / \gamma, u=k r / \gamma$, and $\alpha_{\mathrm{TM}}$ is the unknown parameter which has to be found by field matching at the layers's boundaries. The total (i.e. resistive-wall plus space-charge) longitudinal impedance is given by

$$
Z_{/ /}(\omega)=-\frac{1}{Q} \int_{-\infty}^{+\infty} d z E_{z}^{(s)}(a, z ; \omega) e^{j k z},
$$

which, using Eqs (14), leads to (with $L$ the length of the resistive object)

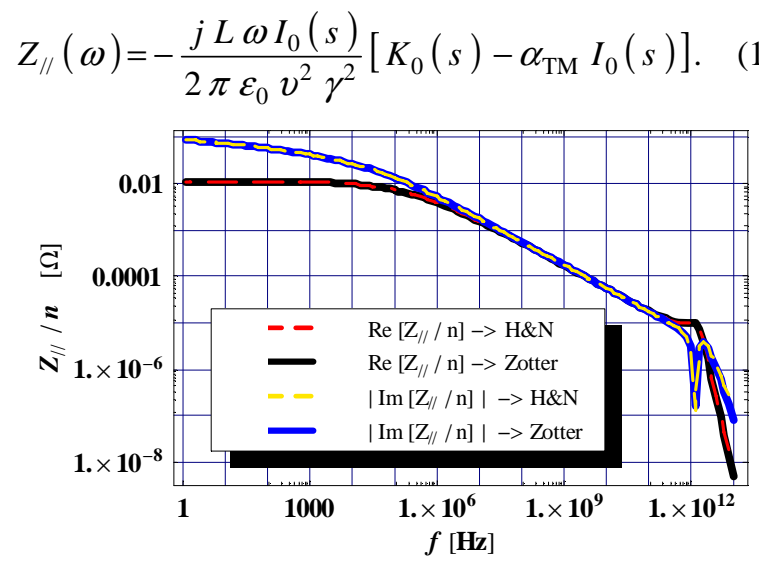

Figure 1: Longitudinal impedance for the case of a (single-layer) $1 \mathrm{~m}$ long (round) LHC graphite collimator with radius $b=2 \mathrm{~mm}$ and conductivity $\sigma=10^{-5} \Omega^{-1} \mathrm{~m}^{-1}$.

The resistive-wall impedance is obtained by subtracting from the total impedance of Eq. (16) the impedance obtained with a perfect conductor at $r=b$, i.e. when 
$\alpha_{\mathrm{TM}}=K_{0}\left(x_{1}\right) / I_{0}\left(x_{1}\right)$ with $x_{1}=k b / \gamma$, which yields the space-charge impedance

$$
Z_{/ /}^{\mathrm{SC}}(\omega)=-\frac{j L \omega I_{0}^{2}(s)}{2 \pi \varepsilon_{0} v^{2} \gamma^{2}}\left[\frac{K_{0}(s)}{I_{0}(s)}-\frac{K_{0}\left(x_{1}\right)}{I_{0}\left(x_{1}\right)}\right] .
$$

The present formalism can be used for any number of layers of the vacuum pipe [13]. Only the result for a single layer extending up to infinity is given here

$$
\begin{aligned}
& Z_{/ /}^{\mathrm{RW}}(\omega)=-\frac{j L \omega I_{0}^{2}(s)}{2 \pi \varepsilon_{0} v^{2} \gamma^{2}} \\
& \times\left[\begin{array}{l}
\frac{K_{0}\left(x_{1}\right)}{I_{0}\left(x_{1}\right)}- \\
\frac{v K_{0}\left(x_{2}\right) K_{0}^{\prime}\left(x_{1}\right)-K_{0}\left(x_{1}\right) K_{0}^{\prime}\left(x_{2}\right) \varepsilon^{\prime} k / \gamma}{v I_{0}^{\prime}\left(x_{1}\right) K_{0}\left(x_{2}\right)-I_{0}\left(x_{1}\right) K_{0}^{\prime}\left(x_{2}\right) \varepsilon^{\prime} k / \gamma}
\end{array}\right],
\end{aligned}
$$

where $x_{2}=v b$. The longitudinal impedance (divided by $\left.n=f / f_{\text {rev }}\right)$ for the case of a LHC collimator is shown in Fig. 1, revealing a good agreement with Ref. [10].

\section{TRANSVERSE IMPEDANCE}

Considering only the term $m=1$ (transverse dipole), the charge density, in the frequency domain, is given by

$$
\rho_{1}(r, \vartheta, z ; \omega)=\frac{P_{1}}{\pi a^{2} v} \delta(r-a) \cos \vartheta e^{-j k z} .
$$

The longitudinal source-field components (for $a \leq r \leq b$ ) have been computed in Ref. [12] and are given by

$$
\begin{aligned}
& E_{z}^{(s)}(r, \vartheta, z ; \omega)=\frac{j \omega P_{1}}{\pi a \varepsilon_{0} v^{2} \gamma^{2}} I_{1}(s) \cos \vartheta \\
& \times\left[K_{1}(u)-\alpha_{\mathrm{TM}} I_{1}(u)\right] e^{-j k z}, \\
& G_{z}^{(s)}(r, \vartheta, z ; \omega)=\frac{j \omega P_{1}}{\pi a \varepsilon_{0} v^{2} \gamma^{2}} I_{1}(s) \sin \vartheta \\
& \times \alpha_{\mathrm{TE}} I_{1}(u) e^{-j k z},
\end{aligned}
$$

where the unknown parameters $\alpha_{\mathrm{TM}}$ and $\alpha_{\mathrm{TE}}$ have to be found by field matching at the layers's boundaries. The total horizontal impedance is given by

$$
\begin{aligned}
& Z_{x}(\omega)=\frac{j}{P_{1}} \int_{-\infty}^{+\infty} d z\left[E_{x}-v_{b} B_{y}\right] e^{j k z} \\
& =\frac{j}{P_{1}} \int_{-\infty}^{+\infty} d z\left[E_{\vartheta}^{(s)}\left(a,-\frac{\pi}{2}, z ; \omega\right)+v_{b} B_{r}^{(s)}\left(a,-\frac{\pi}{2}, z ; \omega\right)\right] e^{j k z}
\end{aligned}
$$

which, using Eqs. (10) and (11), yields

$$
Z_{x}(\omega)=-\frac{j L Z_{0} I_{1}(s)}{\pi a^{2} \beta \gamma^{2}}\left[K_{1}(s)-\alpha_{\mathrm{TM}} I_{1}(s)\right] .
$$

The space-charge impedance is obtained with a perfect conductor at $r=b$, i.e. when $\alpha_{\mathrm{TM}}=K_{1}\left(x_{1}\right) / I_{1}\left(x_{1}\right)$, and is given by

$$
Z_{x}^{\mathrm{SC}}(\omega)=-\frac{j L Z_{0} I_{1}^{2}(s)}{\pi a^{2} \beta \gamma^{2}}\left[\frac{K_{1}(s)}{I_{1}(s)}-\frac{K_{1}\left(x_{1}\right)}{I_{1}\left(x_{1}\right)}\right] .
$$

The present formalism can also be used for any number of layers of the vacuum pipe [7]. Only the result for a single layer extending up to infinity is given here

$$
\begin{aligned}
& Z_{x}^{\mathrm{RW}}(\omega)=j L Z_{0} \beta I_{1}^{2}(s) K_{1}\left(x_{1}\right) x_{1}^{2} x_{2}^{2} \gamma v\left(\frac{I_{1}^{\prime}\left(x_{1}\right)}{I_{1}\left(x_{1}\right)}-\frac{K_{1}^{\prime}\left(x_{1}\right)}{K_{1}\left(x_{1}\right)}\right) \\
& \times\left(\gamma v \frac{I_{1}^{\prime}\left(x_{1}\right)}{I_{1}\left(x_{1}\right)}-k \mu^{\prime} \frac{K_{1}^{\prime}\left(x_{2}\right)}{K_{1}\left(x_{2}\right)}\right) /
\end{aligned}
$$

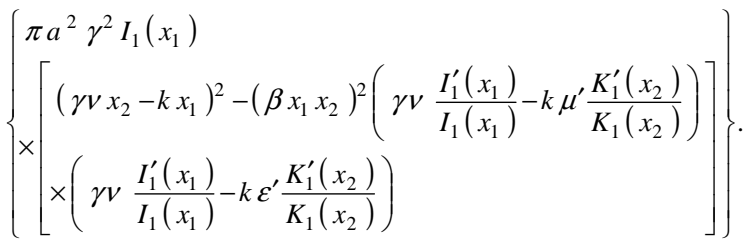

The transverse impedance of a LHC graphite collimator is shown in Fig. 2, in good agreement with Ref. [10].

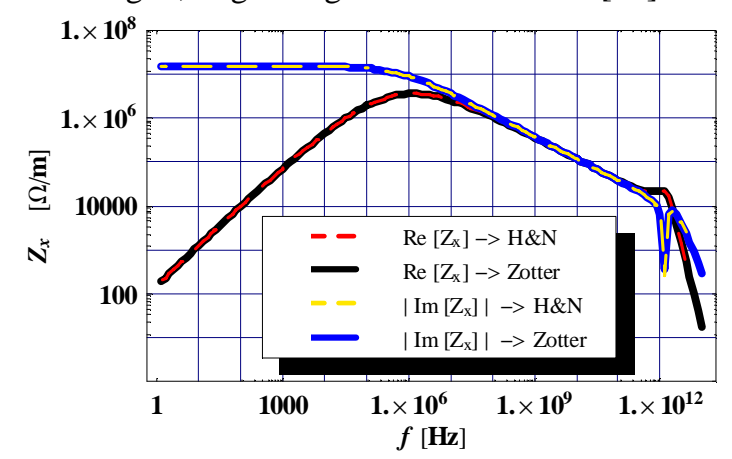

Figure 2: Transverse impedance for the case of a LHC graphite collimator (see Fig. 1).

\section{CONCLUSION}

A general formalism to compute both longitudinal and transverse impedances of an infinitely long multi-layer circular beam pipe has been presented. Three frequency regimes are found [8], in agreement with Ref. [10] in the particular case of a (single-layer) LHC collimator. Comparisons with Ref. [2] can be found in Refs. [7,8].

\section{REFERENCES}

[1] J. Laslett, K. Neil, A. Sessler, Rev. Sci. Instr. (1965).

[2] A. Burov et al., Proc. EPAC’02, Paris, 2002.

[3] H. Tsutsui, LHC Project Note 318, 2003.

[4] L.Vos, CERN-AB-2003-005 ABP, 2003.

[5] F. Caspers et al., Proc. EPAC'04, Lucerne, 2004.

[6] Al-Khateeb et al., Proc. EPAC’06, Edinburgh, 2006.

[7] B. Zotter, CERN-AB-2005-043, 2005.

[8] E. Métral, CERN-AB-2005-084, 2005.

[9] E. Métral, Proc. PAC'2005, Knoxville, 2005.

[10] Henry and Napoly, CLIC Note 142-1991, 1991.

[11] T. Suzuki, KEK Preprint 98-127, 1998.

[12] R.L. Gluckstern, CERN 2000-011, 2000.

[13] B. Salvant et al., to be published. 